\title{
STOCHASTIC DYNAMICS OF TWO PICOPHYTOPLANKTON POPULATIONS IN A REAL MARINE ECOSYSTEM*
}

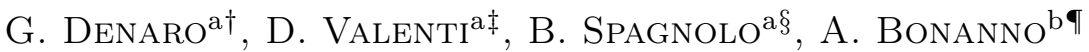 \\ G. Basilone ${ }^{\mathrm{b}}$, S. Mazzola ${ }^{\mathrm{b}}$, S.W. ZGGOzi ${ }^{\mathrm{c}}$, S. AronicA ${ }^{\mathrm{b}}$ \\ ${ }^{a}$ Dipartimento di Fisica e Chimica, Group of Interdisciplinary Physics \\ Università di Palermo and CNISM - Unità di Palermo \\ Viale delle Scienze, Edificio 18, 90128 Palermo, Italy \\ bIstituto per l'Ambiente Marino Costiero, CNR, U.O.S. di Capo Granitola \\ Via del Faro 3, 91020 Campobello di Mazara (TP), Italy \\ ${ }^{\mathrm{c}}$ Marine Biology Research Centre, P.O. Box 30830, Tajura, Libya
}

(Received May 10, 2013)

A stochastic reaction-diffusion-taxis model is analyzed to get the stationary distribution along water column of two species of picophytoplankton, that is picoeukaryotes and Prochlorococcus. The model is valid for weakly mixed waters, typical of the Mediterranean Sea. External random fluctuations are considered by adding a multiplicative Gaussian noise to the dynamical equation of the nutrient concentration. The statistical tests show that shape and magnitude of the theoretical concentration profile exhibit a good agreement with the experimental findings. Finally, we study the effects of seasonal variations on picophytoplankton groups, including an oscillating term in the auxiliary equation for the light intensity.

DOI:10.5506/APhysPolB.44.977

PACS numbers: 05.10.-a, 05.40.-a, 87.23.Cc

\section{Introduction}

In an ecological context, the study of vertical distributions of the picophytoplankton communities is very important to predict and understand future changes in marine ecosystems, produced by global warming [1, 2]. In re-

* Presented at the XXV Marian Smoluchowski Symposium on Statistical Physics, "Fluctuation Relations in Nonequilibrium Regime", Kraków, Poland, September 10-13, 2012.

${ }^{\dagger}$ giovanni.denaro@unipa.it

$¥$ davide.valenti@unipa.it

$\S$ bernardo.spagnolo@unipa.it

I angelo.bonanno@iamc.cnr.it 
cent works, the spatio-temporal dynamics of phytoplankton in the Mediterranean Sea have been studied by using a stochastic approach to describe and simulate the experimental findings for the chlorophyll concentration [3, 4]. In order to analyze the dynamics of phytoplankton, it is worth underlining that marine ecosystems are complex systems, that is open systems characterized by nonlinear interactions between their parts and external perturbations, both deterministic and random, due to environmental variables [5-25]. As a consequence, the study of a marine ecosystem has to be performed by considering also the effects of random perturbations, which can be treated as environmental noise sources. The model is used to analyze the behaviour of two picophytoplankton groups, which are subject to a stochastic dynamics. In particular, in order to better simulate this nonlinear and noisy dynamics, we take into account in our model the presence of external random perturbations by including, in the equation that describes the dynamics of the nutrient concentration, a term of multiplicative noise [3, 4, 12].

In order to analyze the picophytoplankton dynamics, we consider an ecosystem which possesses the hydrological characteristics typical of the Strait of Sicily and is known to be a biologically rich area of the Mediterranean Sea with a key role in terms of fisheries [26-29]. Moreover, the two picophytoplankton groups considered belong to the smaller size fraction (less than $3 \mu \mathrm{m}$ ) of phytoplankton and are mainly represented by picoprokaryotes and picoeukaryotes. Specifically, the picoprokaryotes group is dominated in the Sicily Channel by a species of cyanobacteria, i.e. Prochlorococcus, while picoeukaryotes group is mainly represented by pelagophytes and prymnesiophytes [30]. In this work, we study the vertical profiles of picoeukaryotes and Prochlorococcus, which account for about $60 \%$ of total chlorophyll on average in the Mediterranean Sea.

The interaction of these populations with the environment occurs through two factors that limit the growth of this aquatic microorganisms: light intensity and nutrient. The light penetrates through the surface of the water and decreases exponentially along the water column. Vice-versa, nutrient concentrations along the water column are characterized by an increasing trend from the sea surface to the benthic layer. The opposite gradients of two limiting factors contribute to select different species, and sometimes groups, along the water column, and determine the biodiversity of the ecosystem [1, 2, 4].

The responses of each picophytoplankton group to environment solicitations depend strongly on the biological and physical parameters [31]. The values of the environmental parameters have been chosen to reproduce the marine ecosystem of the Sicily Channel in summer, characterized by oligotrophic water and high light intensity. Biological parameters depend on both the metabolism and cell structure of the specific microorganisms investigated. It is important noting that some biological parameters play a key 
role on the position and magnitude of planktonic groups. In fact, the halfsaturation constants locate the position of the production layer and depth of concentration peak for each picophytoplankton group. Moreover, the growth rates and nutrient uptake determine the balance of the primary production of phytoplankton in marine ecosystem [32], contributing to modify the composition and magnitude of the planktonic communities.

The first goal has been to obtain the spatio-temporal distributions of two groups of picophytoplankton, i.e. picoeukaryotes and Prochlorococcus, using a reaction-diffusion-taxis model [1,2]. As a second step, we studied the spatio-temporal evolution of the biomass concentrations of both groups, obtaining the distributions of the total chlorophyll a (chl a) and divinil chlorophyll a (Dvchl a) concentrations in stationary regime, and comparing the numerical results with experimental data collected in a site of the Sicily Channel. Finally, we analyzed the role of seasonal variations on picophytoplankton dynamics, including an oscillating term in the auxiliary equation for the light intensity.

\section{The model}

The distributions of the two picophytoplankton groups in stationary regime are obtained by using a stochastic model with conditions typical of the Mediterranean Sea, where the vertical water columns are weakly mixed. The dynamics, competition and structuring of phytoplankton groups in marine ecosystems have been studied in a series of theoretical works based on deterministic models [1, 2, 33-35]. In particular, it was shown that biological and physical parameters determine the position of the maximum of the biomass concentration, that can be localized at the surface or, alternatively, in a deep layer. Moreover, these parameters have also to guarantee the coexistence of the two groups [2, 35], i.e. picoeukaryotes and Prochlorococcus, in the deep chlorophyll maximum (DCM), according to experimental findings. In order to take into account all these conditions and describe correctly the picophytoplankton dynamics, we devised a stochastic model by including, in a deterministic advection-reaction-diffusion model [1, 35], a source of the multiplicative Gaussian noise.

\subsection{The stochastic model}

In this paragraph, we use a stochastic model to study the dynamics of the two picophytoplanktonic populations [1, 2] distributed along a onedimensional spatial domain (z-direction). In particular, we assume that the interaction of these microorganisms with the marine environment occurs through the two factors which limit the growth of the planktonic communities: light intensity $(I)$ and nutrient $(R)$, i.e. phosphorus. The stochastic 
model has been obtained by modifying a deterministic model based on a system of three differential equations [1, 2]. The first two equations describe the dynamics of the picophytoplankton groups, i.e. picoeukaryotes $\left(b_{1}(z, t)\right)$ and Prochlorococcus $\left(b_{2}(z, t)\right)$. The third equation simulates the change of nutrient concentration $(R(z, t))$ along the water column.

It is worth noting that the magnitude of the picophytoplankton biomass at depth $z$ depends on three processes: growth, loss, and movement. In our model, we consider these contributes in the differential equations for the dynamics of picophytoplankton groups. Moreover, we also take into account random fluctuations and their effect on the phytoplankton dynamics, by inserting in the equation for the nutrient dynamics a term of spatially uncorrelated noise. In particular, we use a source of multiplicative white Gaussian noise $\xi_{R}(z, t)$ with intensity $\sigma_{R}$ and statistical properties: $\left\langle\xi_{R}(z, t)\right\rangle=0$ and $\left\langle\xi_{R}(z, t) \xi_{R}\left(z^{\prime}, t^{\prime}\right)\right\rangle=\sigma_{R} \delta\left(z-z^{\prime}\right) \delta\left(t-t^{\prime}\right)$. Finally, the light intensity $I(z, t)$ is modeled by a function varying, along the water column, with the depth and biomass concentration. The stochastic model is defined by the following equations

$$
\begin{aligned}
& \frac{\partial b_{1}(z, t)}{\partial t}=b_{1} \min \left(f_{I_{1}}(I), f_{R_{1}}(R)\right)-m_{1} b_{1}+D \frac{\partial^{2} b_{1}(z, t)}{\partial z^{2}}-v_{1} \frac{\partial b_{1}(z, t)}{\partial z} \\
& \frac{\partial b_{2}(z, t)}{\partial t}=b_{2} \min \left(f_{I_{2}}(I), f_{R_{2}}(R)\right)-m_{2} b_{2}+D \frac{\partial^{2} b_{2}(z, t)}{\partial z^{2}}-v_{2} \frac{\partial b_{2}(z, t)}{\partial z} \text {, } \\
& \frac{\partial R(z, t)}{\partial t}=-\sum \frac{b_{i}(z, t)}{Y_{i}} \times \min \left(f_{I_{i}}(I), f_{R_{i}}(R)\right)+D \frac{\partial^{2} R(z, t)}{\partial z^{2}} \\
& +\sum \varepsilon_{i} m_{i} \frac{b_{i}(z, t)}{Y_{i}}+R \xi_{R}(z, t) \\
& I(z)=I_{\text {in }} \exp \left\{-\int_{0}^{z}\left[\sum a_{i} b_{i}(Z)+a_{\mathrm{bg}}\right] d Z\right\},
\end{aligned}
$$

where $v_{1}$ and $v_{2}$ are the buoyancy velocities of the two picophytoplankton groups, i.e. picoeukaryotes and Prochlorococcus, respectively; $D$ is the vertical diffusion coefficient; $\varepsilon_{i}, m_{i}$ and $1 / Y_{i}$ are nutrient recycling coefficient, specific loss rate and nutrient content of the $i$ th picophytoplankton group, respectively; $a_{1}$ and $a_{2}$ are the absorption coefficients of the two picophytoplankton groups, and $a_{\mathrm{bg}}$ is the background turbidity; $I_{\text {in }}$ is the incident light intensity at the water surface. Furthermore, $f_{I_{i}}(I)$ and $f_{R_{i}}(R)$ are given by the Michaelis-Menten formulas

$$
\begin{aligned}
f_{I_{i}}(I) & =r_{i} I /\left(I+K_{I_{i}}\right), \\
f_{R_{i}}(R) & =r_{i} R /\left(R+K_{R_{i}}\right) .
\end{aligned}
$$


Here, $r_{i}$ is the maximum growth rate, $K_{I_{i}}$ and $K_{R_{i}}$ are the half-saturation constants for light intensity and nutrient concentration, respectively, of the $i$ th picophytoplankton group. These constants depend on the metabolism of the specific picophytoplankton groups considered. In particular, the halfsaturation constants, $K_{R_{i}}$ and $K_{I_{i}}$, contribute to determining the position along the water column of the biomass production layer for each group.

The stochastic model also includes six equations for the boundary conditions. In particular, we can observe that phytoplankton biomass does not enter or leave the water column. Therefore, the boundary conditions for concentrations of picoeukaryotes and Prochlorococcus biomass account for the absence of flux through both surface layer $z=0$ and seabed $z=z_{\mathrm{b}}$

$$
\left.\left[D \frac{\partial b_{i}}{\partial z}-v_{i} b_{i}\right]\right|_{z=0}=\left.\left[D \frac{\partial b_{i}}{\partial z}-v_{i} b_{i}\right]\right|_{z=z_{\mathrm{b}}}=0 .
$$

Moreover, we fix the boundary conditions for nutrients, which do not come from the top of the water column but are provided from the bottom. In particular, nutrient concentration at the bottom of the water column, $R\left(z_{\mathrm{b}}\right)$, is set at value $R_{\text {in }}$, which depends on the site investigated. These boundary conditions are described by the following equations

$$
\left.\frac{\partial R}{\partial z}\right|_{z=0}=0, \quad R\left(z_{\mathrm{b}}\right)=R_{\mathrm{in}} .
$$

Equations (1)-(8) form the stochastic reaction-diffusion-taxis model used in this work. By solving them, we obtain the spatio-temporal dynamics of the biomass concentrations of picoeukaryotes and Prochlorococcus, nutrient concentration and light intensity.

\subsection{Biological and environmental parameters}

In order to reproduce the experimental profile of chl $a$ and Dvchl a concentration, measured in a marine site close to the Libyan continental shelf (site L1129b in Fig. 1), we set the values of the environmental and biological parameters so that the presence of a deep chlorophyll maximum for both picophytoplankton groups is guaranteed $[1,2,4]$. According to our previous discussion, the values of the biological parameters have been fixed to simulate the behaviour of picoeukaryotes and Prochlorococcus. In particular, the half-saturation constants for each group are chosen to obtain suitable positions for both the production layers and the peak of biomass concentration. 


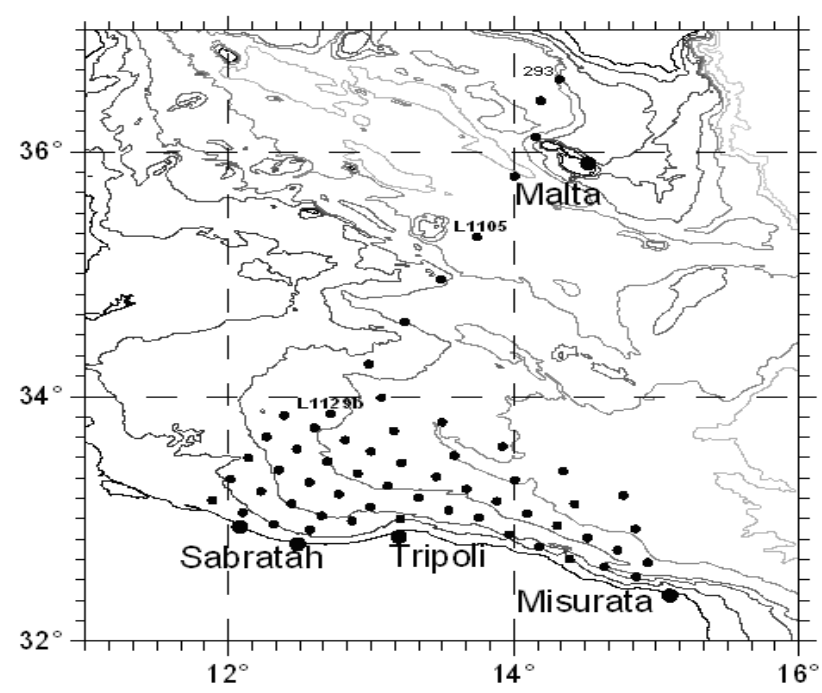

Fig. 1. Locations of marine sites, where experimental data were collected in the period of the $12^{\text {th }}-24^{\text {th }}$ August 2006 in the Sicily Channel area, during the MedSudMed-06 Oceanographic Survey onboard the R/V Urania. Data used in this work, for comparison with theoretical results, are those acquired in site L1129b (close to the Libyan coast).

The parameters $1 / Y_{1}$ and $1 / Y_{2}$ contribute to determine the steady distributions of the picophytoplankton concentrations. In particular, experimental findings show that (i) the peak of biomass concentration of Prochlorococcus is shallower than that of picoeukaryotes and (ii) the average cell concentration of Prochlorococcus along the water column is much higher than that of picoeukaryotes [30,37]. In these conditions, a smaller amount of nutrient is available for Prochlorococcus localized in the biomass peak. Therefore, in order to obtain, for the two picophytoplankton groups, cell concentrations in agreement with the real data, $1 / Y_{2}$ is set at a value much smaller than $1 / Y_{1}$ (see Table I). Moreover, the absorption coefficient of Prochlorococcus, fixed in our model, has to be very different from that of the picoeukaryotes. In fact, due to the higher average cell concentration of Prochlorococcus $\left(5.2 \times 10^{4}\right.$

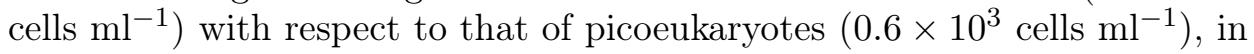
order to simulate the same gradient of light intensity inside the production layers [35], we had to exploit an absorption coefficient for Prochlorococcus lower than that used for picoeukaryotes, setting $a_{2}=2.4 \times 10^{-15} \mathrm{~m}^{2}$ cell $^{-1}$. 
The sinking velocity is set to a value typical of the picophytoplankton [1], while the maximum specific growth rates are in agreement with those experimentally observed by other authors [38]. The values of the environmental parameters have been chosen to reproduce marine ecosystem of the Sicily Channel in summer, i.e. oligotrophic water $\left(R_{\text {in }}<10.0 \mathrm{mmol}\right.$ nutrient $\left.\mathrm{m}^{-3}\right)$ and high light intensity $\left(I_{\text {in }}>1000 \mu \mathrm{mol}\right.$ photons $\left.\mathrm{m}^{-2} \mathrm{~s}^{-1}\right)$. Moreover, the vertical turbulent diffusivity is fixed at values typical of weakly mixed waters $\left(D=1.0 \mathrm{~cm}^{2} \mathrm{~s}^{-1}\right)$. The numerical values of the parameters are reported in Table I.

TABLE I

Parameters used in the stochastic model. The values of the biological parameters are those typical of picophytoplankton groups.

\begin{tabular}{|c|c|c|c|}
\hline Symbol & Interpretation & Units & Site L1129b \\
\hline$I_{\text {in }}$ & Incident light intensity & $\mu \mathrm{mol}$ photons $\mathrm{m}^{-2} \mathrm{~s}^{-1}$ & 1404.44 \\
\hline$a_{\mathrm{bg}}$ & Background turbidity & $\mathrm{m}^{-1}$ & 0.045 \\
\hline$a_{1}$ & Absorption coefficient of picoeukaryotes & $\mathrm{m}^{2}$ cell $^{-1}$ & $6 \times 10^{-10}$ \\
\hline$a_{2}$ & Absorption coefficient of Prochlorococcus & $\mathrm{m}^{2}$ cell $^{-1}$ & $2.4 \times 10-15$ \\
\hline$z_{\mathrm{b}}$ & Depth of the water column & $\mathrm{m}$ & 186 \\
\hline$D$ & Vertical turbulent diffusivity & $\mathrm{cm}^{2} \mathrm{~s}^{-1}$ & 1.0 \\
\hline$r_{1}$ & Maximum specific growth rate & & \\
\hline$r_{2}$ & of $\mathrm{p}$ & $h^{-}$ & 0.08 \\
\hline$r_{2}$ & of Prochlorococcus & $h^{-1}$ & 0.07 \\
\hline$K_{I_{1}}$ & $\begin{array}{l}\text { Half-saturation constant of light-limited } \\
\text { growth of picoeukaryotes }\end{array}$ & $\mu \mathrm{mol}$ photons $\mathrm{m}^{-2} \mathrm{~s}^{-1}$ & 20 \\
\hline$K_{R_{1}}$ & $\begin{array}{l}\text { Half-saturation constant of nutrient-limited } \\
\text { growth of picoeukaryotes }\end{array}$ & mmol nutrient $\mathrm{m}^{-3}$ & 0.0425 \\
\hline$K_{I_{2}}$ & $\begin{array}{l}\text { Half-saturation constant of light-limited } \\
\text { growth of Prochlorococcus }\end{array}$ & $\mu \mathrm{mol}$ photons $\mathrm{m}^{-2} \mathrm{~s}^{-1}$ & 98 \\
\hline$K_{R_{2}}$ & $\begin{array}{l}\text { Half-saturation constant of nutrient-limited } \\
\text { growth of Prochlorococcus }\end{array}$ & mmol nutrient $\mathrm{m}^{-3}$ & 0.0150 \\
\hline$m_{1}$ & Specific loss rate of picoeukaryotes & $h_{1}$ & 0.01 \\
\hline$m_{2}$ & Specific loss rate of Prochlorococcus & $h_{1}$ & 0.01 \\
\hline $1 / Y_{1}$ & Nutrient content of picoeukaryotes & mmol nutrient cell ${ }^{-1}$ & $1 \times 10^{-9}$ \\
\hline $1 / Y_{2}$ & Nutrient content of Prochlorococcus & mmol nutrient cell ${ }^{-1}$ & $4 \times 10^{-15}$ \\
\hline$\epsilon_{1}$ & $\begin{array}{l}\text { Nutrient recycling coefficient } \\
\text { of picoeukaryotes }\end{array}$ & dimensionless & 0.5 \\
\hline$\epsilon_{2}$ & $\begin{array}{l}\text { Nutrient recycling coefficient } \\
\text { of Prochlorococcus }\end{array}$ & 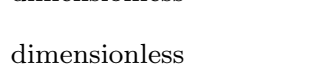 & 0. \\
\hline$v_{1}$ & Buoyancy velocity of picoeukar & $\mathrm{m}$ & -0.0042 \\
\hline$v_{2}$ & Buoyancy velocity of Prochlorococcus & $\mathrm{m} h^{-1}$ & -0.0042 \\
\hline$R_{\text {in }}$ & Nutrient concentration at $z_{\mathrm{b}}$ & mmol nutrient $\mathrm{m}^{-3}$ & 5.0 \\
\hline
\end{tabular}

\subsection{Results of the stochastic model}

In order to obtain the theoretical distributions of biomass concentrations for the two picophytoplankton populations, we solve numerically Eqs. (1)-(8) by using the Ito scheme, and averaging over 1000 realizations [12, 36]. 
The numerical method, whose computer implementation consists in a $\mathrm{C}++$ program, is based on an explicit finite difference scheme with centered-inspace differencing for the diffusion term and upwind differencing for the taxis term. In order to get the steady spatial distributions, we integrate our system over a time interval long enough to obtain the stationary solution. In particular, we solve the equations fixing as a maximum time $t_{\max }=4 \times 10^{4} \mathrm{~h}$. As initial conditions, we set that the picoeukaryotes and Prochlorococcus biomass are concentrated in two layers close to the deep chlorophyll maximum experimentally observed. Moreover, we impose that the nutrient concentration remains approximately constant from the water surface up to the DCM, while increases linearly below the DCM up to the seabed.

We recall that the stochastic model provides the steady distributions of the picophytoplankton concentrations expressed in cell $/ \mathrm{m}^{3}$, while the experimental data of chl a concentrations are expressed in $\mu \mathrm{g} / \mathrm{l}$. Therefore, in order to compare numerical results with the experimental profile, the theoretical cell concentrations of picoeukaryotes and Prochlorococcus are converted into chl $a$ and Dvchl a concentrations, respectively, by using the curves of mean vertical profile (see Fig. 2) obtained by Brunet et al. [37]. In particular, these curves describe the exponential behaviour of the chlorophyll per cell ratio, as a function of depth, for both picophytoplankton groups.
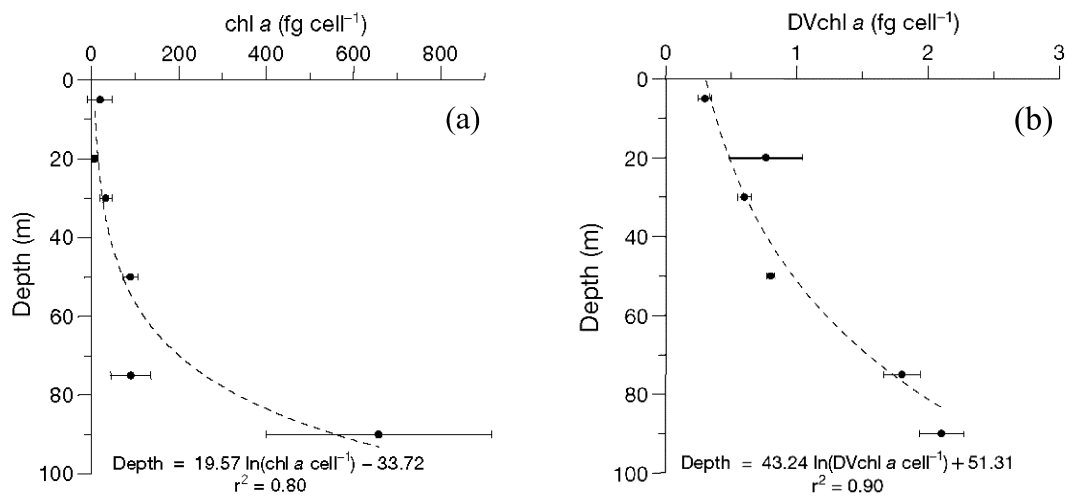

Fig. 2. Mean vertical profile of chl a per picoeukaryote cell (panel a) and Dvchl a per Prochlorococcus cell (panel b). Error bars are Standard Deviation. Equation and $r^{2}$ for the fit are reported on the plots. (Courtesy of Brunet et al. [37].)

Since the structure of the chlorophyll a molecule is almost identical to that of divinil chlorophyll a, we summed their concentrations to obtain theoretical steady profile consistent with those obtained from the experimental data. Moreover, in the Mediterranean Sea about $43 \%$ of the total quantity of chl $a$ and divinil chl $a[1,30]$ is due to nano- and micro-phytoplankton, 
and Synechococcus. Therefore, we consider this fraction of the total biomass and divide it by depth, obtaining for each site the value $\Delta b_{(D v) c h l a}$, which represents a constant concentration of chl $a$ and Dvchl a due to other phytoplankton species present in the water column. Finally, we add the theoretical concentrations with $\Delta b_{(D v) c h l a}$. By this way, we obtain the stationary distributions in deterministic regime and for three different values of the noise intensity. The results are shown in Fig. 3. Here, we observe that the noise causes a decrease and a deeper localization of the DCM. Position, shape and magnitude of the phytoplankton peak, obtained by the stochastic model, exhibit the best agreement with those of the experimental DCM for a noise intensity equal to 0.0040 . This result is confirmed by the reduced
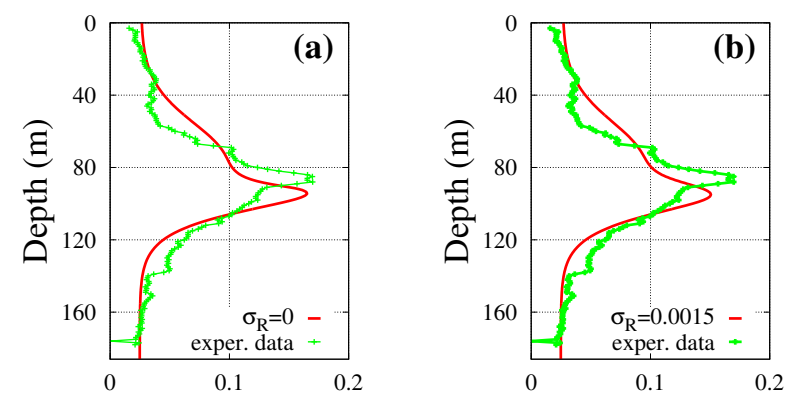

Chl a + Dvchl a conc. $(\mu \mathrm{g} / \mathrm{l}) \quad$ Chl a + Dvchl a conc. $(\mu \mathrm{g} / \mathrm{l})$
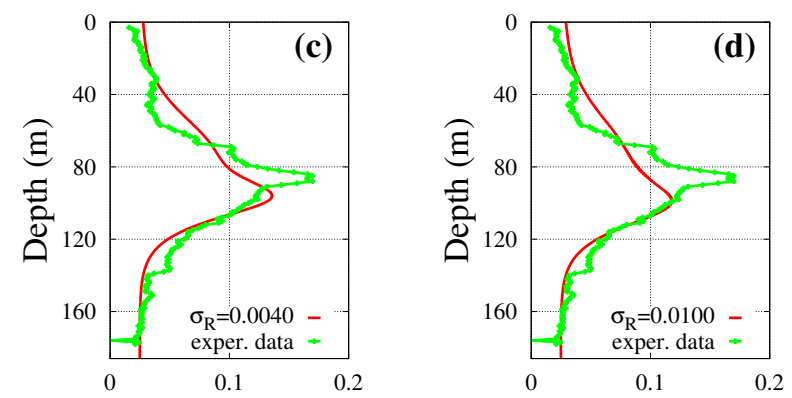

Chl a + Dvchl a conc. $(\mu \mathrm{g} / \mathrm{l})$ Chl a + Dvchl a conc. $(\mu \mathrm{g} / \mathrm{l})$

Fig. 3. Theoretical distributions (gray/red line) of the total chl a and Dvchl $a$ concentration calculated for different values of $\sigma_{R}$ by the stochastic model as a function of depth. The results are compared with the distributions of the total chl $a$ and Dvchl a concentration measured (light gray/green line) in site L1129b. The theoretical values were obtained averaging over 1000 numerical realizations. The values of the parameters are those shown in Table I. The noise intensities are: (a) $\sigma_{R}=0$ (deterministic case), (b) $\sigma_{R}=0.0015$, (c) $\sigma_{R}=0.0040$ and (d) $\sigma_{R}=0.0100$. 
$\chi^{2}$ test that provides the best value, $\tilde{\chi}^{2}=0.0037$, for the same value of noise intensity (see Table II). In particular, the values of the reduced chisquare resulted to be much lower than the value previously obtained by the one-species model [4].

Other results (here not shown) reveal a rapid disappearance of phytoplankton biomass for $\sigma_{R}>0.01$. This indicates that the stability of the nutrient concentration is a critical factor for both picophytoplankton populations studied in this paper. Finally, these results suggest that random fluctuations of the nutrient concentration could be the cause of the collapse of phytoplankton biomass in real marine ecosystems.

TABLE II

Results of $\chi^{2}$ and reduced chi-square $\left(\tilde{\chi}^{2}\right)$ goodness-of-fit test for site L1129b at different values of $\sigma_{R}$. The number of samples along the water column is $n=176$.

\begin{tabular}{cccc}
\hline \hline$R_{\text {in }}$ & $\sigma_{R}$ & $\chi^{2}$ & $\tilde{\chi}^{2}$ \\
\hline 5 & 0.0000 & 0.74 & 0.0042 \\
5 & 0.0015 & 0.67 & 0.0039 \\
5 & 0.0040 & 0.65 & 0.0037 \\
5 & 0.0100 & 0.78 & 0.0045
\end{tabular}

\section{Picophytoplankton dynamics and changes of DCM in the presence of periodical driving force}

In this section, we study the time behaviour of the picophytoplankton groups in presence of seasonal variations of light intensity. In particular, in order to predict the seasonal variations of the primary production of phytoplankton in the Sicily Channel, we insert in our model an oscillating term in the auxiliary equation for the light intensity.

Recent theoretical works [3] indicate that the seasonal variations of light intensity have a strong effect on the distributions of phytoplankton populations along the water column. Therefore, in order to better reproduce spatio-temporal dynamics of picophytoplankton groups, we consider the parameter $I_{\text {in }}$ as a periodical function of time. As a consequence, we replace in Eq. (4) the incident light intensity with the following equation

$$
I_{\text {in }}(t)=I_{\text {in }}^{\text {aver }}+I_{0} \cos \omega t,
$$

where, $I_{\mathrm{in}}^{\text {aver }}$ is the yearly weighted average of the light intensity on the sea surface taking into account only sunny days, i.e. in absence of cloud coverage, and $I_{0}$ is a multiplicative term that describes the magnitude of the seasonal oscillations of the incident light intensity. 

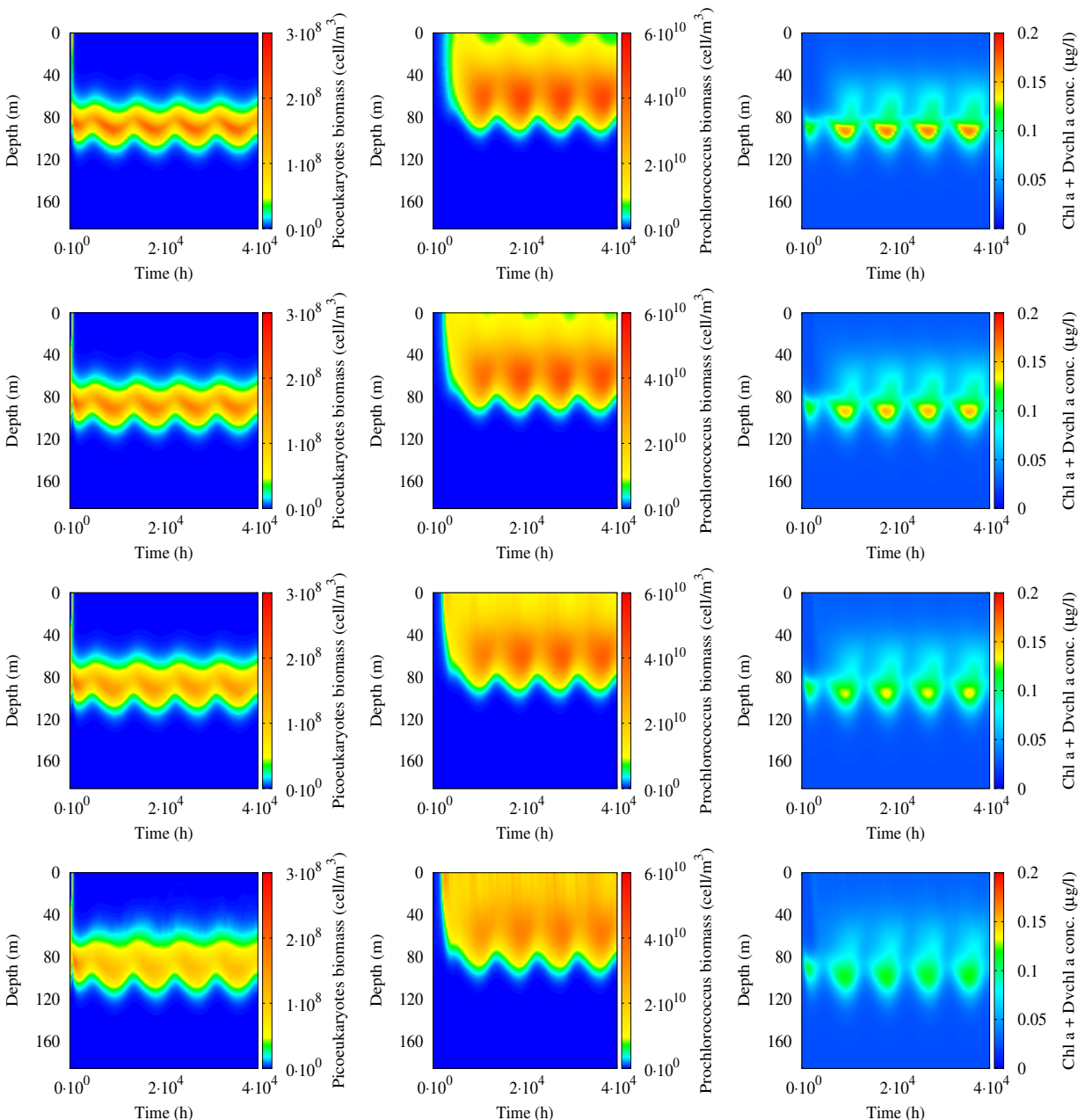

Fig.4. Spatio-temporal behaviour of picoeukaryotes biomass, Prochlorococcus biomass and total $c h l a$ and Dvchl a concentration (from left to right). Contour maps were obtained in the presence of periodical (seasonal) behaviour of the light intensity $I_{\text {in }}(t)$ according to Eq. (9), with the nutrient concentration subject to random fluctuations (see Eq. (3)). The results refers to different values of the noise intensity: $\sigma_{R}=0$ (deterministic regime), $\sigma_{R}=0.0015, \sigma_{R}=0.0040$ and $\sigma_{R}=0.0100$ (from top to bottom). All contour maps were obtained averaging over 1000 numerical realizations. The average value of the incident light intensity is $I_{\text {in }}^{\text {aver }}=1068.58 \mu \mathrm{mol}$ photons $\mathrm{m}^{-2} \mathrm{~s}^{-1}$. The values of the other parameters are those shown in Table I. 
We analyze the ecosystem dynamics in the presence of oscillating light intensity and random fluctuations of environmental variables. In particular, as a first step, we obtain the spatio-temporal dynamics of picoeukaryotes and Prochlorococcus by solving equation system (1)-(8). Second, as in previous section, we convert the theoretical cell concentrations of the picophytoplankton groups into chl $a$ and Dvchl a concentrations, by using the curves of mean vertical profile shown in Fig. 2. The spatio-temporal dynamics of the two picophytoplankton groups and total chl $a$ and Dvchl a concentration is shown in Fig. 4. Here, we observe a decreasing magnitude of the picophytoplankton concentrations during autumn, followed by an increase of biomass concentrations in spring. Moreover, in winter, we obtain a width of the DCM less than that of the summer period. Finally, we note that the position of picophytoplankton concentration peaks changes during the whole solar year, due to the seasonal variations of incident light intensity.

We recall that the position, magnitude and shape of phytoplankton distributions depend on three environmental parameters: the incident light intensity at the sea surface, the nutrient concentration at the seabed and the vertical turbulent diffusivity along water column. In particular, since the seasonal variations influence the values of the incident light intensity, the dynamics of picophytoplankton groups can pass from deep chlorophyll maximum to upper chlorophyll maximum (UCM) stability and vice-versa [2]. This behaviour occurs in marine ecosystems, such that analyzed in this work, close to a border between eutrophic and oligotrophic waters. Moreover, the vertical turbulent diffusivity could change due to random modifications of the environmental variables, i.e. velocity field, salinity and temperature, inducing a faster transition from one chlorophyll concentration profile to another $[2,35]$. In our study, we observe also that the Prochlorococcus concentration reaches higher values close to the water surface, as the noise intensity increases, with the system approaching the UCM configuration (see Fig. 4). A stochastic model, where seasonal variations are considered, allows therefore to take into account more possible scenarios for the spatio-temporal dynamics of phytoplankton biomass in real marine ecosystems.

\section{Conclusions}

In this work, we studied a stochastic model for the spatio-temporal dynamics of two picophytoplankton populations, i.e. picoeukaryotes and Prochlococcus, which tend to occupy different zones of the water column. In particular, the model was devised to investigate the picophytoplankton dynamics in a site of the Sicily Channel, where the waters are prevalently oligotrophic and the climatic parameters are those typical of a temperate region. In order to better reproduce the experimental profile of the total chl a 
and Dvchl a concentration, we took in account the effects of the random perturbations due to environmental variables.

The numerical results showed that the presence of a noise source, which acts directly on the dynamics of the nutrient, allows to simulate the average stationary profile of the total chl $a$ and Dvchl $a$ concentration, obtaining a better agreement with the experimental findings respect to the deterministic case. In particular, the $\chi^{2}$ goodness-of-fit test indicated that the position and magnitude of the DCM are coherent with those of the real data. In addition, the results obtained agree with experimental data reported in Refs. [30, 37]. Finally, we observed that the external random perturbations can give rise to two interesting effects on phytoplankton dynamics: (i) "shift" of the peak of biomass concentrations towards a greater depth; (ii) "disappearance" of picoeukaryotes and Prochlorococcus for higher noise intensity.

We completed our study by analyzing the role of the seasonal oscillations of the light intensity, simulating the spatio-temporal dynamics of the picophytoplankton concentrations during the solar year. The numerical results obtained in the presence of periodical driving force could be useful to predict and understand future changes in the phytoplankton profiles in the Mediterranean Sea, contributing to prevent in marine ecosystem the decline of the phytoplankton production and the consequent decrease of fish species [2, 39-41].

\section{REFERENCES}

[1] J. Huisman, N.N. Pham Thi, D.M. Karl, B. Sommeijer, Nature 439, 322 (2006).

[2] A.B. Ryabov, L. Rudolf, B. Blasius, J. Theor. Biol. 263, 120 (2010).

[3] D. Valenti et al., Acta Phys. Pol. B 43, 1227 (2012).

[4] G. Denaro et al., Ecol. Complex. 13, 21 (2013).

[5] B.T. Grenfell et al., Nature 394, 674 (1998).

[6] C. Zimmer, Science 284, 83 (1999).

[7] O.N. Bjørnstad, B.T. Grenfell, Science 293, 638 (2001).

[8] B. Spagnolo, M. Cirone, A. La Barbera, F. de Pasquale, J. Phys. Condens. Mat. 14, 2247 (2002).

[9] A. La Barbera, B. Spagnolo, Physica A 314, 120 (2002).

[10] B. Spagnolo, A. La Barbera, Physica A 315, 114 (2002).

[11] B. Spagnolo, A. Fiasconaro, D. Valenti, Fluct. Noise Lett. 3, L177 (2003).

[12] B. Spagnolo, D. Valenti, A. Fiasconaro, Math. Biosci. Eng. 1, 185 (2004).

[13] B. Spagnolo, D. Valenti, A. Fiasconaro, Prog. Theor. Phys. Supp. 157, 312 (2005).

[14] A. Caruso et al., Fluct. Noise Lett. 5, L349 (2005). 
[15] O. Chichigina, D. Valenti, B. Spagnolo, Fluct. Noise Lett. 5, L243 (2005).

[16] A. Fiasconaro, D. Valenti, B. Spagnolo, Eur. Phys. J. B50, 189 (2006).

[17] D. Valenti, L. Schimansky-Geier, X. Sailer, B. Spagnolo, Eur. Phys. J. B50, 199 (2006).

[18] O.A. Chichigina, Eur. Phys. J. B65, 347 (2008).

[19] A. La Cognata, D. Valenti, A. Dubkov, B. Spagnolo, Phys. Rev. E81, 011121 (2010).

[20] O.A. Chichigina, A. Dubkov, D. Valenti, B. Spagnolo, Phys. Rev. E84, 021134 (2011).

[21] D. Valenti, A. Fiasconaro, B. Spagnolo, Acta Phys. Pol. B 35, 1481 (2004).

[22] A. Fiasconaro, D. Valenti, B. Spagnolo, Acta Phys. Pol. B 35, 1491 (2004).

[23] G. Bonanno, D. Valenti, B. Spagnolo, Eur. Phys. J. B53, 405 (2006).

[24] D. Valenti, B. Spagnolo, G. Bonanno, Physica A 382, 311 (2007).

[25] D. Valenti et al., Ecol. Model. 213, 449 (2008).

[26] J. Garcia Lafuente et al., Fish Oceanogr. 11, 31 (2002).

[27] A. Cuttitta et al., Hydrobiologia 503, 117 (2003).

[28] M. Ribera d'Alcalà, G. Civitarese, F. Conversano, R. Lavezza, J. Geophys. Res. 108, 8106 (2003).

[29] B. Patti et al., Sci. Mar. 74, 577 (2010).

[30] C. Brunet et al., Aquat. Microb. Ecol. 44, 127 (2006).

[31] J. Norberg, Limnol. Oceanogr. 49, 1269 (2004).

[32] B.B. Prézelin, M.M. Tilzer, O. Schofield, C. Haese, Aquat. Sci. 53, 136 (1991).

[33] J. Huisman, F.J. Weissing, Am. Nat. 146, 536 (1995).

[34] C.A. Klausmeier, E. Litchman, S.A. Levin, J. Theor. Biol. 246, 278 (2007).

[35] A.B. Ryabov, Theor. Ecol. 5, 373 (2012).

[36] A. Giuffrida et al., Eur. Food Res. Technol. 228, 767 (2009).

[37] C. Brunet, R. Casotti, V. Vantrepotte, F. Conversano, Mar. Ecol. Prog. Ser. 346, 15 (2007).

[38] C. Dimier, C. Brunet, R. Geider, J. Raven, Limnol. Oceanogr. 54, 823 (2009).

[39] L. Bopp et al., Global Biogeochem. Cy. 15, 81 (2001).

[40] J.L. Sarmiento et al., Global Biogeochem. Cy. 18, GB3003 (2004).

[41] A. Schmittner, Nature 434, 628 (2005). 